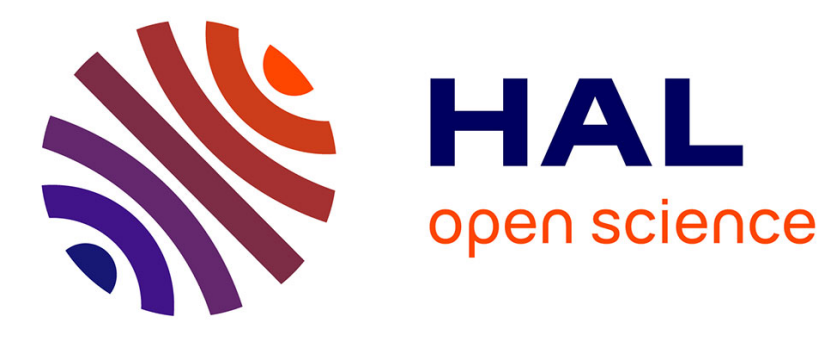

\title{
Topological band crossings in epitaxial strained SnTe
}

\author{
Sotirios Fragkos, Roberto Sant, Carlos Alvarez, Evangelos Golias, Jose
}

Marquez-Velasco, Polychronis Tsipas, Dimitra Tsoutsou, Sigiava

Aminalragia-Giamini, Evangelia Xenogiannopoulou, Hanako Okuno, et al.

\section{To cite this version:}

Sotirios Fragkos, Roberto Sant, Carlos Alvarez, Evangelos Golias, Jose Marquez-Velasco, et al.. Topological band crossings in epitaxial strained SnTe. Physical Review Materials, 2019, 3 (10), pp.104201. 10.1103/PhysRevMaterials.3.104201 . hal-02491506

\section{HAL Id: hal-02491506 https://hal.science/hal-02491506}

Submitted on 26 Feb 2020

HAL is a multi-disciplinary open access archive for the deposit and dissemination of scientific research documents, whether they are published or not. The documents may come from teaching and research institutions in France or abroad, or from public or private research centers.
L'archive ouverte pluridisciplinaire HAL, est destinée au dépôt et à la diffusion de documents scientifiques de niveau recherche, publiés ou non, émanant des établissements d'enseignement et de recherche français ou étrangers, des laboratoires publics ou privés. 


\title{
Topological band crossings in epitaxial strained SnTe
}

\author{
Sotirios Fragkos $\odot,{ }^{1,2}$ Roberto Sant,${ }^{3,4}$ Carlos Alvarez $\odot,{ }^{3,5}$ Evangelos Golias $\odot,{ }^{6,7}$ Jose Marquez-Velasco $\odot,{ }^{1}$ \\ Polychronis Tsipas $\odot,{ }^{1}$ Dimitra Tsoutsou, ${ }^{1}$ Sigiava Aminalragia-Giamini $\odot,{ }^{1}$ Evangelia Xenogiannopoulou $\odot,{ }^{1}$ \\ Hanako Okuno, ${ }^{3,5}$ Gilles Renaud, ${ }^{3,5}$ Oliver Rader, ${ }^{6}$ and Athanasios Dimoulas $\oplus^{1, *}$ \\ ${ }^{1}$ Institute of Nanoscience and Nanotechnology, National Center for Scientific Research "Demokritos," 15310 Athens, Greece \\ ${ }^{2}$ Department of Mechanical Engineering, University of West Attica, 12244 Athens, Greece \\ ${ }^{3}$ Université of Grenoble Alpes, 38400 Grenoble, France \\ ${ }^{4}$ Neel Institute, Centre National de la Recherche Scientifique, 38042 Grenoble, France \\ ${ }^{5}$ Interdisciplinary Institute of Research of Grenoble, Commissariat à l'Énergie Atomique et aux Énergies Alternatives, \\ 38054 Grenoble, France \\ ${ }^{6}$ Helmholtz-Zentrum Berlin für Materialien und Energie, Elektronenspeicherring BESSY II, \\ Albert-Einstein Straße 15, 12489 Berlin, Germany \\ ${ }^{7}$ Institut für Experimentalphysik, Freie Universität Berlin, Arnimallee 14, 14195 Berlin, Germany
}

(Received 24 July 2019; revised manuscript received 3 September 2019; published 14 October 2019)

\begin{abstract}
Epitaxial $\mathrm{SnTe}(111)$ is grown by molecular-beam epitaxy on $\mathrm{Bi}_{2} \mathrm{Te}_{3}$ substrates. Structural evaluation indicates that SnTe deviates from cubic due to in-plane compressive strain, which induces significant changes in the electronic band structure. More specifically, a pair of gapless crossings between the two uppermost valence bands occurs in $k$ space along the out-of-plane $\Gamma Z$ direction of the Brillouin zone, associated with a band inversion, thus defining topological three-dimensional Dirac nodes. Combined first-principles calculations and angle-resolved photoelectron spectroscopy reveal an overtilted Dirac cone indicating that the crossing is a topological type-III Dirac node at the borders between type-I and type-II Dirac nodes.
\end{abstract}

DOI: 10.1103/PhysRevMaterials.3.104201

\section{INTRODUCTION}

Theoretical predictions and experimental evidence show that $\mathrm{SnTe}$ and related compounds could have a rich spectrum of physical properties, namely, a giant bulk Rashba effect coupled with ferroelectricity and nontrivial topological order [1-6]. In more detail it has been predicted [3] that SnTe could be found in a number of different phases ranging from a topological crystalline insulator (TCI) and the timereversal invariant $Z_{2}$ topological insulator to a ferroelectric (FE) Rashba semiconductor depending on the parameter of distortion $\lambda$ from the cubic $(F m \overline{3} m)$ phase $c$-SnTe $(\lambda=0)$ to a rhombohedral $(R 3 m)$ noncentrosymmetric $r$-SnTe $(\lambda=1)$ phase.

At room temperature, the most stable bulk phase is cubic while other phases like $r$-SnTe become stable at low temperatures, therefore many interesting properties as those mentioned above cannot be easily investigated or exploited in practical applications. On the other hand, epitaxial (ultra)thin films, especially along the polar (111)-oriented surface, offer an opportunity to stabilize metastable phases either due to epitaxial strain or due to size effects. Strain can cause rhombohedral distortions with broken inversion symmetry leading to ferroelectricity. It has been recently demonstrated [7] that twodimensional SnTe ultrathin (two to eight atomic layers) films can be grown in an orthorhombic structure which is ferroelectric with in-plane polarization. Moreover, magnetotransport

*Corresponding author: a.dimoulas@inn.demokritos.gr measurements on epitaxial SnTe (111) TCI indicate Rashba splitting of bulk bands [8], originating from the breaking of inversion symmetry as the structure deviates from cubic.

Apart from symmetry-breaking structural distortions as described above, substrate-driven epitaxial strain can modify the electronic bands in a nontrivial way. The topic of band crossings at a single point in $k$ space induced by strain is of immense interest at present. In semimetals, unavoided conduction- and valence-band crossings at a single point in $k$ space, associated with band inversion, often lead to 3D Dirac-like cones with nontrivial topology hosting Dirac or Weyl fermions [9]. Particularly interesting classes are the topological type-III Dirac and Weyl semimetals with exciting predicted analogies with cosmology [10-14]. A large number of exotic topological materials have been predicted [15-17], igniting an intense effort to prove them experimentally. Interestingly, bulk $\mathrm{Pb}_{1-x} \mathrm{Sn}_{x} \mathrm{Te}$, a material similar to $\mathrm{SnTe}$, becomes a Weyl semimetal in a pressure range of 15-25 kbars [4]. Apparently, SnTe thin films deserve more attention to investigate the possibility of nontrivial band crossings that could occur at ambient pressure as a result of epitaxial stress from the substrate.

There are several works reporting the molecular-beam epitaxy (MBE) growth of SnTe (111) on different substrates such as $\mathrm{BaF}_{2}$ (111) [18,19] and $\mathrm{Bi}_{2} \mathrm{Te}_{3}$ [20,21]. The growth on $\mathrm{Bi}_{2} \mathrm{Te}_{3}$ templates is particularly interesting due to nearly matching conditions. Angle-resolved photoemission spectroscopy (ARPES) measurements of the SnTe (111) surface $[21,22]$ reveal Dirac conelike surface states at the $\bar{\Gamma}$ and $\bar{M}$ points of the surface Brillouin zone (BZ) associated with band 
inversion at the $\mathrm{L}$ point of the bulk $\mathrm{BZ}$ which is compatible with the known TCI character of $c$-SnTe. The bulk valence band is also observed peaking at $\bar{\Gamma}[21,22]$ near the Fermi level, which corresponds to the $\mathrm{L}$ point of the bulk BZ, in agreement with theoretical predictions of the $c$-SnTe (111) surface $[3,23]$. In addition to the surface Dirac cones at the $\bar{\Gamma}$ and $\bar{M}$ points, an unidentified Dirac conelike bulk band structure has been observed when the photon energy crosses the $\Gamma$ point of the bulk BZ, the Dirac point of which is well below the Fermi level [19].

In the present paper, 50 units of SnTe (111) film are grown by $\mathrm{MBE}$ on four quintuple-layer (QL) $\mathrm{Bi}_{2} \mathrm{Te}_{3} / \mathrm{AlN}(0001) / \mathrm{Si}(111)$ substrates. A detailed analysis by high-resolution synchrotron grazing incidence $\mathrm{x}$-ray diffraction (GIXRD) and by cross-sectional scanning transmission electron microscopy (STEM) indicates that $\mathrm{SnTe}$ is epitaxially deposited on the $\mathrm{Bi}_{2} \mathrm{Te}_{3}$ template. The data indicate an in-plane compressive strain in the (111) SnTe films resulting in an out-of-plane expansion along the [111] direction. By using first-principles calculations and the experimentally determined lattice parameters, we found that this structural change creates a pair of 3D Dirac crossings between the two uppermost valence bands around $1.83 \mathrm{eV}$ below Fermi level symmetrically placed with respect to the $\Gamma$ point along the $\Gamma \mathrm{Z}\left(k_{z}\right)$ direction of the BZ. The $\Gamma \mathrm{Z}$ direction, which is in general inequivalent to the $\Gamma \mathrm{L}$ $\left(k_{x, y}\right)$ direction, arises from an anisotropy of the BZ due to the out-of-plane expansion, while it becomes equivalent for cubic structure [1,2,5,19-23]. One of these bands remains dispersionless along $k_{z}$ for some part of $k$ space, while the other one disperses upwards crossing the flat band, thus realizing a type-III Dirac node, existing only as a theoretical possibility up to now. We further confirm the predictions from first-principles calculations by imaging this type of crossing at $\bar{\Gamma}$ by synchrotron-based ARPES.

\section{EPITAXIAL GROWTH}

The substrates consisting of 200-nm epitaxial wurtzite AlN(0001) layers on $\mathrm{Si}(111)$ by metal organic chemical vapor deposition are chemically cleaned in hydrogen fluoride (HF) followed by in situ ultrahigh vacuum (UHV) thermal annealing at $700{ }^{\circ} \mathrm{C}$ for $15 \mathrm{~min}$ prior to growth. The tellurides growth is performed in an $\mathrm{MBE}$ chamber. The $\mathrm{Bi}_{2} \mathrm{Te}_{3}$ buffer layer is first grown on $\mathrm{AlN}(0001) / \mathrm{Si}(111)$ substrate, followed by $\mathrm{SnTe}$ growth without breaking the vacuum. $\mathrm{Sn}$ is evaporated using an electron-gun evaporator while $\mathrm{Bi}$ and $\mathrm{Te}$ are evaporated from effusion cells heated at 600 and $320^{\circ} \mathrm{C}$, respectively. $\mathrm{Bi}_{2} \mathrm{Te}_{3}$ is grown at $300^{\circ} \mathrm{C}$, becoming unstable at higher temperatures under UHV conditions; therefore, SnTe growth temperature is also limited to $300{ }^{\circ} \mathrm{C}$. The Te/Sn growth rates are maintained at a ratio $1: 1$, although Te overpressure can also be applied without changing the SnTe stoichiometry.

The in situ reflection high-energy electron-diffraction (RHEED) patterns of AlN substrate, $\mathrm{Bi}_{2} \mathrm{Te}_{3}$, and $\mathrm{SnTe}$ are shown in Fig. 1, indicating a good in-plane alignment between the layers and the substrate along crystallographic dimensions of high symmetry. The relative positions of SnTe and $\mathrm{Bi}_{2} \mathrm{Te}_{3}$ diffraction streaks indicate that the SnTe lattice constant is slightly larger than that of $\mathrm{Bi}_{2} \mathrm{Te}_{3}$. Assuming that
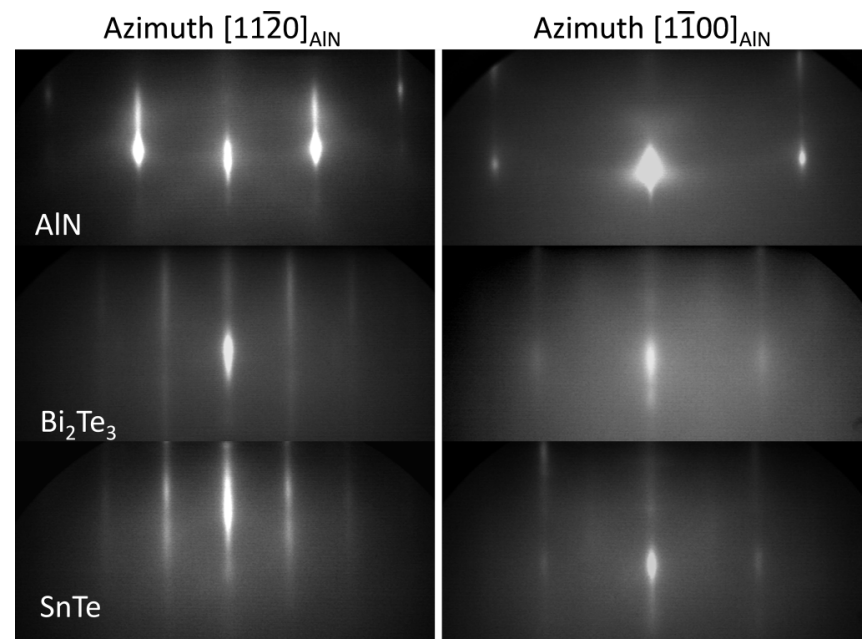

FIG. 1. RHEED diffraction patterns of the AlN substrate and the epilayers along the two nonequivalent high-symmetry directions of AlN.

$a(\mathrm{AlN})=3.11 \AA$ [24], from RHEED the lattice constants $a\left(\mathrm{Bi}_{2} \mathrm{Te}_{3}\right)$ and $a(\mathrm{SnTe})$ are estimated to be 4.38 and 4.44 $\AA$, respectively. While $a\left(\mathrm{Bi}_{2} \mathrm{Te}_{3}\right)$ agrees with reported values from the literature and with density functional theory (DFT) calculations, $a(\mathrm{SnTe})$ deviates slightly from known $c$-SnTe and $r$-SnTe values, which are $4.462 \AA$ [25] and $4.467 \AA$ [26], respectively.

\section{STRUCTURAL CHARACTERIZATION}

\section{A. Cross-sectional scanning transmission electron microscopy}

Film morphology and structure were observed in cross section by STEM. The cross-sectional specimen was prepared by a focused ion beam (FIB) and immediately inserted into the electron microscope to avoid oxidation of the films. STEM experiments were carried out at $200 \mathrm{kV}$ on a Cs-corrected Field electron and ion company (FEI) Themis equipped with a Super-X detector for energy dispersive X-ray analysis. Highangle annular dark-field (HAADF) STEM images were acquired using a convergence semiangle of $18 \mathrm{mrad}$, and collecting scattering $>65 \mathrm{mrad}$. Nanobeam precession diffraction (NPED) patterns were acquired using a convergence semiangle of $2.4 \mathrm{mrad}$, precession angle of $0.3 \mathrm{deg}$, and precessed frequency of $200 \mathrm{~Hz}$. STEM specimens were prepared by the FIB lift-out technique using a FEI dual-beam Strata $400 \mathrm{~S}$ at $30 \mathrm{kV}$.

A cross-sectional HAADF image of the SnTe layer on the $\mathrm{Bi}_{2} \mathrm{Te}_{3}$ buffer along the SnTe [321] zone axis is shown in Fig. 2(a), with the corresponding fast Fourier-transform (FFT) pattern and the simulated diffraction pattern in Figs. 2(b) and 2(c), respectively. In the HAADF image of the SnTe film, the horizontal layer by layer contrast variation corresponds to the $\mathrm{Sn}$ and Te layers in the SnTe structure. Below the SnTe layer is the $\mathrm{Bi}_{2} \mathrm{Te}_{3}$ buffer layer expected from the growth. The $\mathrm{Bi}_{2} \mathrm{Te}_{3}$ layer consists of three full quintuples and an additional half cell of the quintuplet. The SnTe layer was additionally analyzed by the FFT pattern (the diffractogram) to locally determine the crystal structure of the SnTe. A simulated 

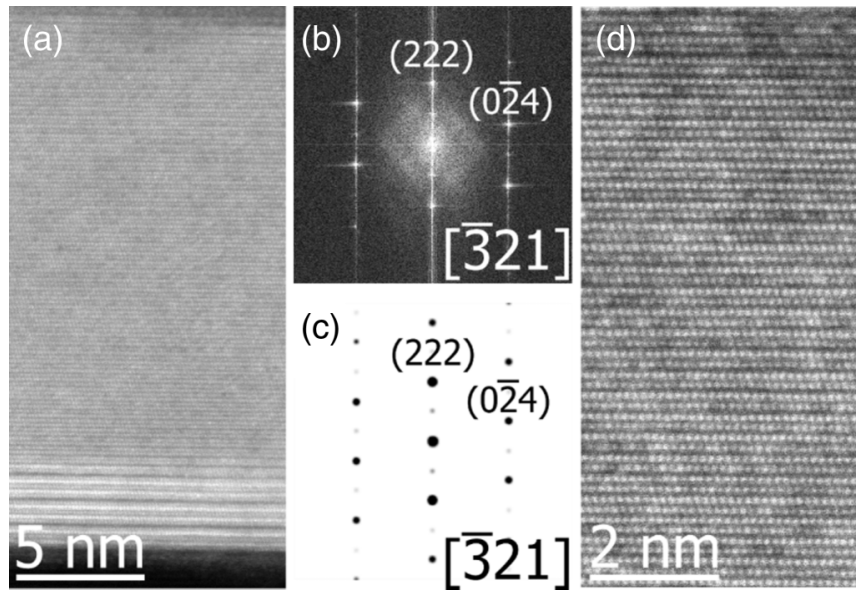

FIG. 2. HAADF image of (a) SnTe film along the [321] zone axis with corresponding (b) fast Fourier transform and (c) simulated diffraction pattern of SnTe along the same zone axis. (d) Highresolution HAADF-STEM image of SnTe film. The layered structure at the bottom of the film in (a) consists of three QLs of $\mathrm{Bi}_{2} \mathrm{Te}_{3}$ buffer.

electron-diffraction pattern for both cubic and rhombohedral

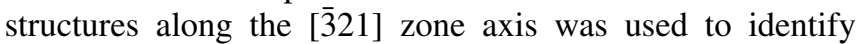
the length and angle ratios between the diffracted spots for each structure. Careful examination of the experimental FFT in comparison with the simulated diffraction patterns shows deviation from the cubic structure.

NPED experiments $[27,28]$ were conducted on the SnTe film to spatially resolve the crystal structure of the SnTe. A NPED pattern, shown in Fig. 3(a), was acquired at each point over the region shown in the STEM image, Fig. 3(b). The NPED data cube map is calibrated with a reference ( $\mathrm{Si}$ substrate). Following calibration, the in-plane and outof-plane reflections of $g(\overline{2} 20)$ and $g(22 \overline{2})$, respectively, are separately used to determine the lattice parameter for the SnTe at each point [Fig. 3(c)]. The resulting lattice parameter

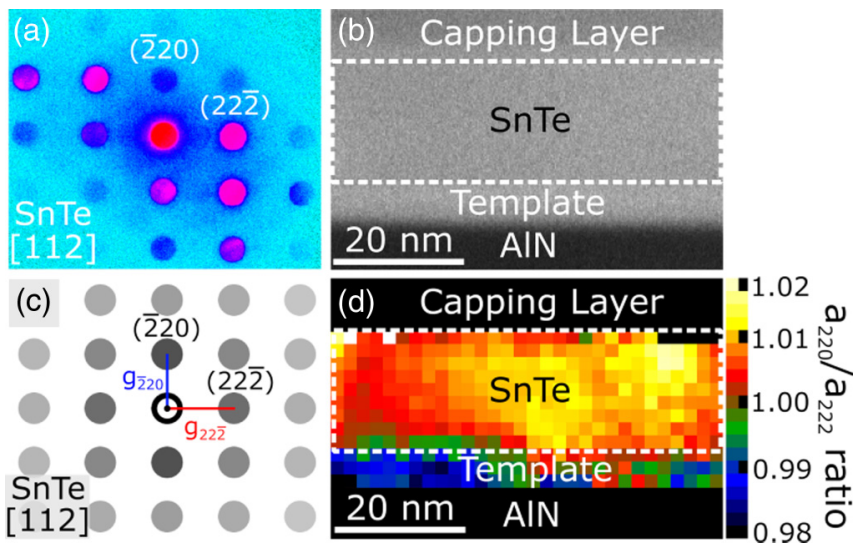

FIG. 3. (a) NPED pattern from the SnTe layer along the [112] zone axis. (b) STEM image of the region analyzed with NPED. (c) Simulated NPED pattern along [112] showing the in-plane reflections $(\overline{2} 20)$ and out-of-plane $(22 \overline{2})$ reflections which were used to determine the ratio of lattice parameter $r=\left(\alpha_{(\overline{2} 20)} / \alpha_{(22 \overline{2})}\right)$. (d) Map of the $r$ ratio in the SnTe region (dashed box), where each pixel corresponds to a NPED pattern.
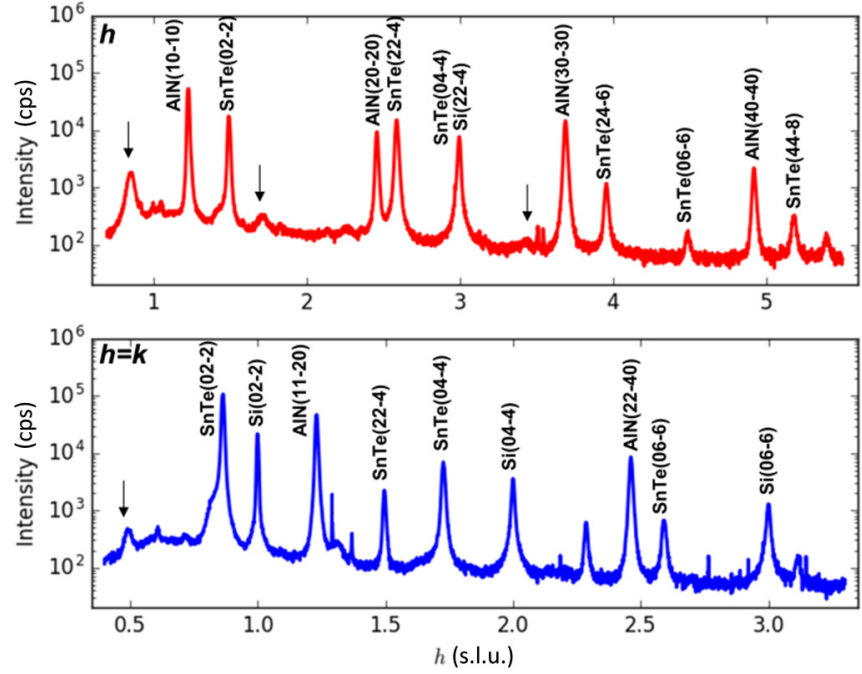

FIG. 4. Radial scans along the in-plane $h$ and $h=k$ directions as a function of the $\mathrm{Si}(111)$ surface reciprocal-lattice units. Each peak has been indexed according to International Centre for Diffraction Data database files using cubic (SnTe, Si) and hexagonal (AlN) Miller index notations. Arrows point out peaks which are not Bragg peaks but overlayer SnTe crystal truncation rod signals. The peak patterns in $h$ and $h=k$ directions are compatible with the presence of $30^{\circ}$ rotated domains.

ratio map $r=\left(\alpha_{(\overline{2} 20)} / \alpha_{(22 \overline{2})}\right)$ is shown in Fig. 3(d), with each pixel representing the ratio determined by a single electrondiffraction pattern. The $r$ ratio for the SnTe film is larger than 1 , indicating a deviation from cubic structure for which case $r$ is expected to be equal to unity. The variation on the map of the $r$ ratio quantitatively is from 1.001 to 1.018 , moving left to right. Probing the entire SnTe region, the average $r$ ratio is 1.009 , with a standard deviation of 0.00318 . It should be noted that in the $\mathrm{Bi}_{2} \mathrm{Te}_{3}$ region $r$ is below 1 due to the difference of crystal structure from the reference and SnTe region, which was not accounted for in the calculation.

\section{B. High-resolution grazing incidence XRD analysis}

GIXRD measurements were performed using the UHVMBE-CVD diffractometer of the BM32 beamline at the European Synchrotron Radiation Facility in Grenoble, France with x-ray energies (wavelengths) of $12.5 \mathrm{keV}(0.9918 \AA$ ). The incident beam, of energy resolution $10^{-4}$, had a very small $\left(5 \times 10^{-5} \mathrm{rad}\right)$ divergence parallel to the surface. The incident angle was set at $0.17^{\circ}$, which is slightly below the critical angle for total external reflection, in order to enhance the thin-film signal while minimizing the background from the AlN buffer layer.

Radial scans were performed along the high-symmetry directions, i.e., $h$ and $h=k$ (Fig. 4), and scans were performed along the rods of scattering perpendicular to the surface on all measurable Bragg peaks.

In Table I all measured in-plane and out-of-plane SnTe peaks are reported with bulk cubic Miller indexing along with reference data for $c$-SnTe [25] and $r$-SnTe [26] for comparison. The $d$ spacings measured for (220) and $(02 \overline{2})$ reflections differ from each other $(2.2438 \AA$ against $2.2240 \AA)$ 
TABLE I. Interplanar distances indexed in SnTe bulk cubic Miller notation for in-plane and out-of-plane data, respectively. $d_{\exp }$ are experimental values deduced in this paper, $d_{\text {calc }}$ are calculated values obtained from a simulated rhombohedral distorted SnTe structure with the experimentally measured lattice parameters, and $d_{\text {ref.cub }}$ and $d_{\text {ref.rh }}$ are taken from SnTe cubic and rhombohedral references, respectively (see text). The $d$ spacings measured for (220) and $(02 \overline{2})$ reflections show the effect of distortion on the $\{220\}$ family, the correspondent distances of which should be equal in a perfect cubic system but split in a distorted one with lower symmetry.

\begin{tabular}{lcccc}
\hline \hline$h k l$ & $d_{\text {exp }}(\AA)$ & $d_{\text {calc }}(\AA)$ & $d_{\text {ref.cub }}(\AA)$ & $d_{\text {ref.rh }}(\AA)$ \\
\hline \multicolumn{5}{c}{ Out of plane } \\
$11 \overline{1}$ & 3.6294 & 3.6353 & 3.6432 & 3.6484 \\
$00 \overline{2}$ & 3.1752 & 3.1559 & 3.1551 & 3.1614 \\
220 & 2.2438 & 2.2398 & 2.2310 & 2.2375 \\
$\overline{1} \overline{13}$ & 1.9062 & 1.9120 & 1.9026 & 1.9086 \\
$13 \overline{1}$ & 1.8929 & 1.9018 & 1.9026 & 1.9061 \\
$11 \overline{3}$ & n.m. & 1.8968 & 1,9026 & 1.9049 \\
$22 \overline{2}$ & 1.8223 & 1.8177 & 1.8216 & 1.8242 \\
$00 \overline{4}$ & 1.5799 & 1.5780 & 1.5776 & 1.5807 \\
$33 \overline{1}$ & n.m. & 1.4497 & 1.4477 & 1.4510 \\
240 & 1.4117 & 1.4155 & 1.4110 & 1.4148 \\
$33 \overline{3}$ & 1.2114 & 1.2118 & 1.2149 & 1.2161 \\
$15 \overline{3}$ & 1.0635 & 1.0640 & 1.0667 & 1.0681 \\
$44 \overline{2}$ & 1,0510 & 1.0519 & 1.0522 & 1.0538 \\
$26 \overline{2}$ & 0.9508 & 0.9509 & 0.9513 & 0.9531 \\
$17 \overline{5}$ & n.m. & 0.7412 & 0.7290 & 0.7295 \\
$28 \overline{4}$ & 0.6875 & 0.6873 & 0.6888 & 0.6895 \\
& \multicolumn{5}{c}{ In plane } & \\
$02 \overline{2}$ & 2.2240 & 2.2235 & 2.2310 & 2.2335 \\
$22 \overline{4}$ & 1.2837 & 1.2837 & 1.2886 & 1.2895 \\
$04 \overline{4}$ & 1.1117 & 1.1118 & 1.1155 & 1.1168 \\
$06 \overline{6}$ & 0.7409 & 0.7412 & 0.7440 & 0.7445 \\
\hline \hline
\end{tabular}

although they are expected to be equivalent by symmetry in a perfect cubic structure. This indicates that epitaxial compressive strain followed by expansion along the growth direction causes SnTe cell distortion and lowers the symmetry, thus alleviating the multiplicity of the equivalent planes as observed.

To deduce SnTe lattice parameters Eq. (1) is used, which is generally valid for hexagonal lattice systems:

$$
\frac{1}{\boldsymbol{d}^{2}}=\frac{4}{3} \frac{\left(\boldsymbol{h}^{2}+\boldsymbol{h} \boldsymbol{k}+\boldsymbol{k}^{2}\right)}{\boldsymbol{a}^{2}}+\frac{\boldsymbol{l}^{2}}{\boldsymbol{c}^{2}}
$$

The same formula, though, can be used to describe a face-centered-cubic lattice grown along the (111) direction by properly choosing a hexagonal basis with in-plane lattice parameter $a$ equal to the side length of the (111) surface unit cell and an out-of-plane parameter $c$ equal to the body diagonal of the cube $D$. Then, by fitting the positions of the in-plane Bragg peaks the interplanar spacings $d$ are obtained and subsequently $a$ is deduced from Eq. (1) by setting $l=0$. The average value of the in-plane lattice parameter was found to be $a=(4.447 \pm 0.001) \AA$, significantly smaller than those calculated from unstrained cubic phase (4.462 ̊) [25] and rhombohedral SnTe (4.467 $\AA$ ) [26] (Table II). It is anticipated that $\mathrm{SnTe}$ is grown under epitaxial in-plane compressive strain
TABLE II. Summary of the results obtained from experimental values and cubic and rhombohedral references; listed from left to right are hexagonal unit-cell lattice constants $(a, c)$ and distortion ratio $(c / D)$, where $D$ is the body diagonal of the pure cubic phase unit cell.

\begin{tabular}{lccc}
\hline \hline & $a(\AA)$ & $c(\AA)$ & $c / D$ \\
\hline Exp. & 4.447 & 11.013 & 1.011 \\
Cubic [25] & 4.462 & 10.929 & 1 \\
Rhomb. [26] & 4.467 & 10.971 & 1.003 \\
\hline \hline
\end{tabular}

to better match the in-plane lattice constant of $4.38 \AA$ of the $\mathrm{Bi}_{2} \mathrm{Te}_{3}$ template.

Subsequently, using the calculated value $a$, and the interplanar distance positions $d$ of the remaining out-of-plane peaks, the lattice parameter $c$ was also estimated from Eq. (1), and an average value $c=(11.013 \pm 0.012) \AA$ was obtained. As already mentioned above, in an ideal cubic lattice oriented along the (111) direction, the body diagonal $D$ is exactly equal to the vertical lattice constant $c$ of the hexagonal cell. Under the assumption that the grown SnTe (111) has an ideal cubic lattice with the measured surface lattice parameter in the (111) plane $a=4.447 \AA$, the cubic unit-cell diagonal should be expected to be $D=a \sqrt{ } 6=10.893 \AA$. This value is smaller than the measured $c$, indicating that SnTe significantly deviates from the ideal cubic structure. In quantitative terms, SnTe shows an out-of-plane expansion characterized by a ratio $c / D=1.011$, larger than unity. Lattice constants and distortion ratios for experimental and reference values are reported in Table II.

The results are in good agreement with the STEM/NPED observations (Sec. II) confirming deviations of epitaxial $\mathrm{SnTe}$ from the cubic structure. Although the XRD and STEM/NPED data cannot confirm a noncentrosymmetric $(R 3 m)$ rhombohedral distortion, they undoubtedly point to a rhombohedral distortion with an appreciable out-of-plain expansion induced by the substrate. This could modify the electronic band structure in a nontrivial way as discussed in the next two sections.

\section{FIRST-PRINCIPLES CALCULATIONS}

Here, the effect of out-of-plane tensile strain in the electronic band structure of SnTe (Fig. 5) is discussed, based on DFT calculations. The first-principles calculations were performed using the Vienna $\mathrm{Ab}$ initio Simulation Package (VASP) $[29,30]$. The generalized-gradient approximation with Perdew-Burke-Ernzerhof [31] parametrization was used as exchange-correlation functional. The kinetic-energy cutoff was set at $500 \mathrm{eV}$ and the reciprocal space was sampled using the Monkhorst-Pack scheme [32] employing a $16 \times$ $16 \times 16 k$-point mesh. Spin-orbit coupling was included for the band-structure calculations. In the case of cubic SnTe, a fourfold degeneracy is observed exactly at the $\Gamma$ point with a quadratic dispersion along all three directions of the Brillouin zone [33]. The 1.1\% out-of-plane tensile strain derived from our structural characterizations pushes the two valence bands into one another, thus creating a pair of unavoided Dirac nodes along the $\Gamma \mathrm{Z}\left(k_{z}\right)$ direction. 


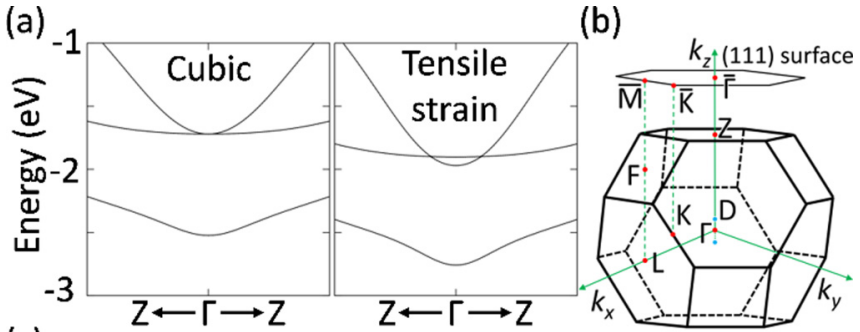

(c)

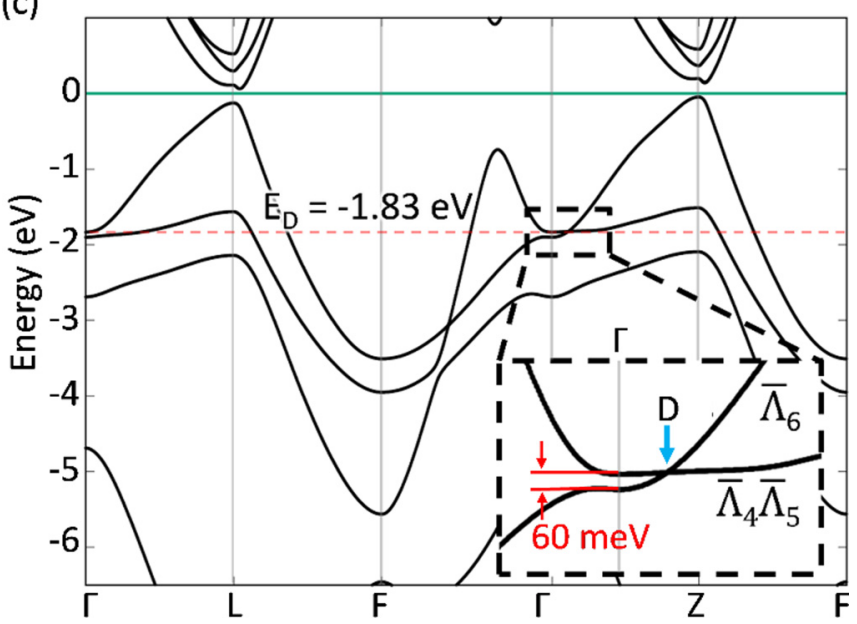

FIG. 5. (a) The evolution of the band structure under out-ofplane tensile strain along the [111] direction along the $\mathrm{Z}-\Gamma-\mathrm{Z}$ direction of (b) the Brillouin zone. Note that $\mathrm{Z}$ and $\mathrm{L}$ points become equivalent in the unstrained cubic case. (c) Band structure of bulk out-of-plane tensile strained SnTe along the $\Gamma$-L-F- $\Gamma-\mathrm{Z}-\mathrm{F}$ direction of the Brillouin zone. The inset shows a magnification around the Dirac point $\mathrm{D}$ marked by the blue vertical arrow.

The calculated bulk band structure of SnTe with the experimental lattice constants (out-of-plane tensile strain along [111]) in Fig. 5(c) reveals unavoided band crossings along the $\Gamma-\mathrm{Z}$ direction of the Brillouin zone between the two uppermost doubly degenerate valence bands at $1.83 \mathrm{eV}$ below Fermi level, which is similar to the case of $\mathrm{PtTe}_{2}$ [34]. VASP2TRACE code [16] was used to examine the topology and calculate the irreducible representations at the high-symmetry points of VASP wave functions. The decompositions of these irreducible representations, as we move along the $\Gamma-\mathrm{Z}$ direction, were deduced from the BANDREP program [35-37] on the Bilbao Crystallographic Server. From these calculations, it is inferred that the gapless crossing occurs because the two bands belong to different irreducible representations $\left(\bar{\Lambda}_{6}\right.$ and $\left.\bar{\Lambda}_{4} \bar{\Lambda}_{5}\right)$ which prohibit hybridization and gap opening. As a result, a pair of fourfold Dirac nodes is generated at $\pm\left(0.053 a^{*}, 0.053 b^{*}\right.$, $0.053 c^{*}$ ) [indicated as D in Fig. 5(a)], which are protected by $C_{3}$ rotational symmetry along the $k_{z}$ axis [38].

The $\bar{\Lambda}_{4} \bar{\Lambda}_{5}$ band is flat near the Dirac point while $\bar{\Lambda}_{6}$ disperses upwards crossing the flat band. This is also observed in the in-plane band dispersions for several "cuts" of the $\Gamma-Z$ direction [Fig. 6(a)] where the second (first) uppermost valence band remains dispersionless in energy at $\bar{\Gamma}$ while the first (second) one is displaced upwards as we move away from $\mathrm{D}$ along $k_{z}$ towards $\mathrm{Z}(\Gamma)$, indicating that the crossing is a typeIII Dirac point. Plotting the band structure in Fig. 6(b), along
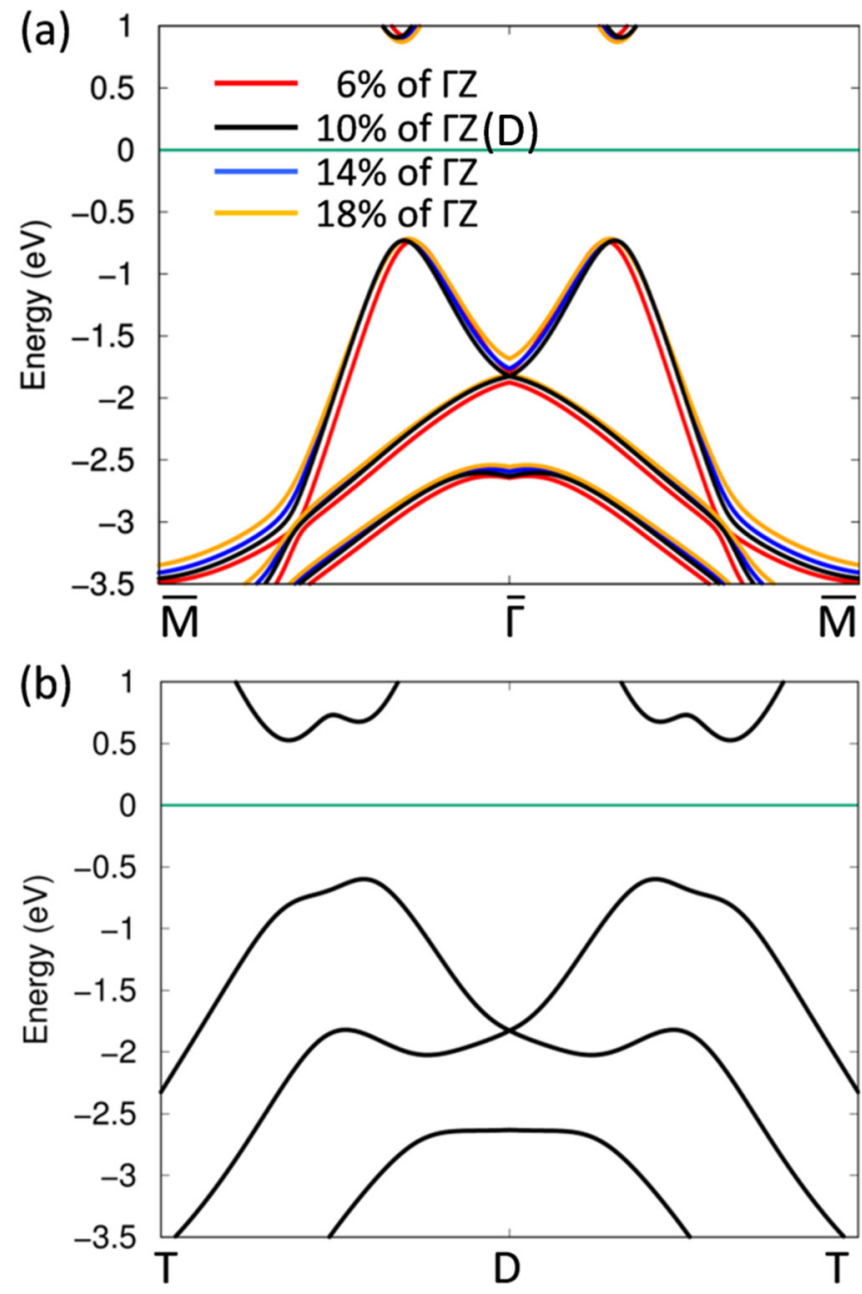

FIG. 6. (a) The in-plane band dispersions at different positions along the $\Gamma-\mathrm{Z}$ direction expressed as relative deviations (\%) from $\Gamma$. At relative deviation of $10 \%$, the in-plane cut passes from the Dirac point $\mathrm{D}$ (black curve) predicting a crossing at D. (b) Band structure along the T-D-T direction, which is parallel to the $\mathrm{K}-\Gamma-\mathrm{K}$ direction. A band crossing at $\mathrm{D}$ is predicted.

a direction T-D-T parallel to $\mathrm{K}-\Gamma-\mathrm{K}$, a crossing is unveiled at the same Dirac point D as in Fig. 6(a). This shows that all bands cross at a single point in $k$ space defining a 3D Dirac node.

\section{ELECTRONIC BAND-STRUCTURE IMAGING BY ARPES}

Specially prepared samples with 100-nm-thick Te capping were measured at the synchrotron facility BESSY II, after capping removal, with horizontally polarized light and variable photon energy in order to follow the electronic band dispersion along the $k_{z}$ axis. The results of synchrotron radiation ARPES data are presented in Fig. 7 of the main text.

The main characteristic is a crossing between the two uppermost valence bands at the center of the surface Brillouin Zone ( $\bar{\Gamma}$ point) around $1.2 \mathrm{eV}$ [Fig. $7(\mathrm{a})]$ below the Fermi level, which is in qualitative agreement with the in-plane band dispersion calculated by DFT [Fig. 6(a)]. There is a difference in the position of the theoretical [Fig. 6(a)] and 


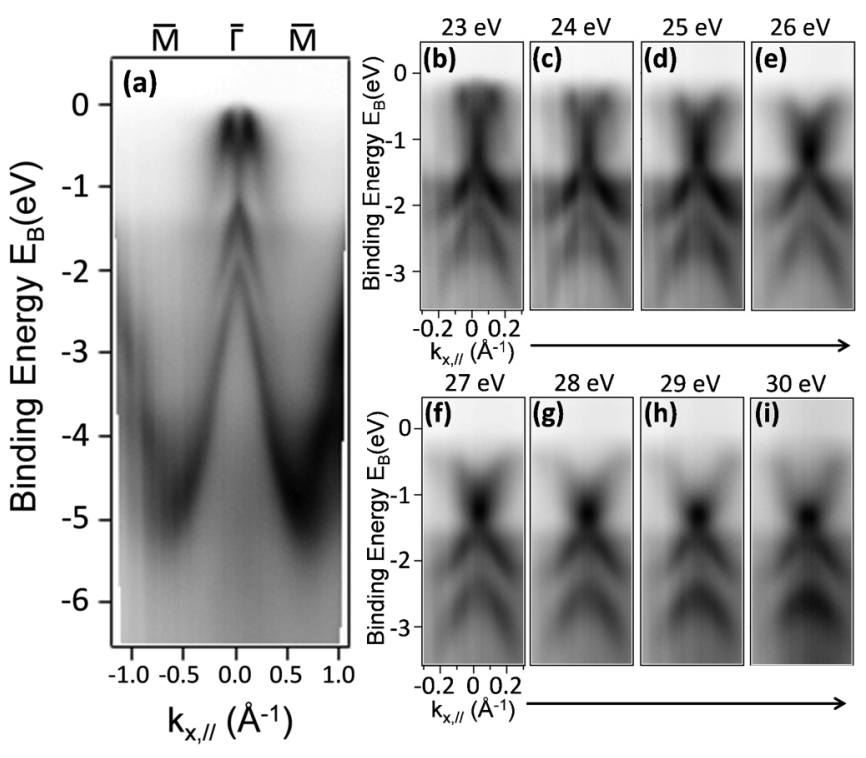

FIG. 7. (a) Energy dispersion along the $\bar{M}-\bar{\Gamma}-\bar{M}$ direction of the Brillouin zone taken with photon energy $h v=65 \mathrm{eV}$ and (b)-(i) ARPES of SnTe taken at different photon energies $h v$ from 23 to $30 \mathrm{eV}$.

experimental [Fig. 7] peaks of the M-shaped band which varies depending on the photon energy [Figs. 7(a) and 7(b)7(i)] or, equivalently, on the out-of-plane $k_{z}$ values of the experimental energy dispersions. Since the theoretical and experimental $k_{z}$ values do not necessarily match, a deviation is expected in the dispersions. For a better and more quantitative comparison between theory and experiment, knowledge of the experimental $k_{z}$ value is required for each photon energy. However, the latter quantity cannot be determined since the inner potential $V_{0}$ is unknown (see also discussion in Sec. VI below). For the same reason, it is not possible to extract the experimental energy dispersion $E$ vs $k_{z}$ to compare with the theoretical dispersion in Fig. 5(c) along the $\Gamma \mathrm{Z}$ direction of the BZ.

Another noticeable difference between the experimental [Fig. 7(a)] and theoretical band structure [Fig. 6(a)] is that for the experimental one the maximum of the M-shaped band around $\bar{\Gamma}$ touches the Fermi level, while in the case of DFT the same band lies $0.6 \mathrm{eV}$ below $E_{F}$. The crossing is also located $0.6 \mathrm{eV}$ above that predicted by DFT. This M-shaped band is analogous to what is observed for $\mathrm{PtTe}_{2}$ near the typeII Dirac cone [34]. Further investigation at different photon energies [Figs. 7(b)-7(i)] reveals that the energy dispersion of the uppermost M-shaped valence band depends strongly on photon energy while the second $\Lambda$-shaped band remains invariant, indicating a dispersionless band along the $\Gamma-Z\left(k_{z}\right)$ direction of the Brillouin zone.

It should be noted that varying photon energy a vertically dispersive feature appears between the upper $\mathbf{M}$ and lower $\Lambda$ bands which is attributed to many-body interactions that cannot be predicted by DFT calculations of the single-particle spectrum as previously reported [39] for the case of $\mathrm{Bi}$ (111) bilayer grown on $\mathrm{Bi}_{2} \mathrm{Te}_{3}$. Given the observations above and theoretical first-principles calculations, the band crossing is interpreted as a type-III 3D Dirac node, which is further discussed in the next section.

\section{DISCUSSION AND CONCLUSIONS}

In this paper, SnTe (111) thin films have been grown on $\mathrm{Bi}_{2} \mathrm{Te}_{3}$ buffer layers by MBE. A combination of synchrotron GIXRD and STEM measurements indicates that the substrate imposes an in-plane compressive strain associated with a corresponding out-of-plane expansion. This deviation from cubic structure causes nontrivial band-structure modifications studied by ARPES and first-principles calculations (DFT).

Room-temperature ARPES measurements of SnTe [Fig. 7] show a characteristic feature which appears as a crossing of two different valence bands at the $\bar{\Gamma}$ point of the surface BZ. Since $k_{z}$ depends on the photon energy and the inner potential $V_{0}$ [19,22], which could be different in samples grown in different conditions, the location of the crossing point along $\Gamma \mathrm{Z}$ of the bulk BZ cannot be easily inferred without prior measurement of $V_{0}$. For example, in $c$-SnTe obtained by bulk growth methods, $V_{0}=6.9 \mathrm{eV}$ [22], and for a photon energy of $23 \mathrm{eV}$ ARPES traces the L point of the BZ, while in epitaxial SnTe with $V_{0}=21.5 \mathrm{eV}$ [19] and for similar photon energies of $25 \mathrm{eV}$ ARPES traces the $\Gamma$ point.

It is worth noting that a similar feature as in Fig. 7 is predicted [3] at the $\mathrm{Z}$ point of the $\mathrm{BZ}$ in noncentrosymmetric $r$-SnTe which has been attributed to the Rashba splitting of the bulk bands, raising questions about the origin of our ARPES observations. Although a noncentrosymmetric, ferroelectric $r$-SnTe is expected to be stable only below $100 \mathrm{~K}$ [40], several works report ferroelectricity in SnTe at room temperature [41] and others provide evidence [42] for rhombohedral distortions persisting up to room temperature in a cubic matrix. Therefore, at first sight a Rashba spitting due to noncentrosymmetric $r$-SnTe cannot be excluded as a possible explanation of our ARPES observations. In such a case though, the Rashba parameter $\alpha_{R}=2 \Delta E_{R} / k_{R}$ from Fig. 7 is estimated to be up to $7 \mathrm{eV} \AA$, that is considerably larger than the theoretically predicted value (4.4 eV $\AA$ ) [3] for the valence band of $r$-SnTe and is also larger than the value of $5 \mathrm{eV} \AA$ reported in prototypical bulk Rashba material BiTeI, suggesting that the explanation of our ARPES observations in terms of Rashba splitting in less probable. Moreover, several other characteristics of the Mshaped feature in Fig. 7 are not compatible with Rashba effect. For example, the crossing point in the ARPES measurements occurs deeper in the valence band while the Rashba crossing inferred from calculations [3] is much closer to the Fermi level. In addition, the $\mathrm{M}$-shaped part of the band above the crossing changes as photon energy varies, while the $\Lambda$-shaped part below the crossing point remains unchanged, which is not compatible with typical Rashba splitting behavior. Although it is tempting to assign the $\Lambda$-shaped band to a surface state known to be invariable under photon energy changes, our DFT calculations [Fig. 5(c)] suggest that the invariance of this band is due to its flat energy dispersion along $\Gamma-Z$ near $\Gamma$.

Following the aforementioned analysis of experimental and theoretical data, it is concluded here that a special type of 3D Dirac node is formed where two valence bands cross at a single point in $k$ space (the Dirac point), located along $\Gamma-Z$ and very close to $\Gamma$. The Dirac node shows linear dispersion at the vicinity of the Dirac point in all three dimensions in $k$ space and a flat dispersion of one of the bands. Materials possessing Dirac nodes are typically Dirac semimetals (DSMs) 
and Weyl semimetals and are categorized either as type-I DSM with untilted or slightly tilted Dirac cone and a pointlike Fermi surface or as type-II DSM where the Dirac cone is overtilted producing electron and hole pockets touching at the Dirac point.

The existence of type-I and type-II Dirac and Weyl crossings has been experimentally confirmed [34,43-50] in several topological semimetals. Yet a type-III crossing emerges as a theoretical possibility exactly at the borders between type-I and type-II crossings, characterized by a unique linelike Fermi surface and a flat energy dispersion along one direction in the BZ. The search for type-III Dirac and Weyl semimetals is partly motivated $[10,11]$ by the spectacular predictions that these materials (i.e., black phosphorous [12], $\mathrm{Zn}_{2} \mathrm{In}_{2} \mathrm{~S}_{5}$ [13], and photonic orbital graphene [14]) could be the solid state (or fermionic) analog of the black hole event horizon potentially generating "Hawking radiation" at relatively high "Hawking temperature" [10]. Except for a recent report related to artificial photonic orbital graphene lattices [14], type-III Dirac nodes have not been experimentally realized in other crystals. Here, we propose strained SnTe as a candidate host of type-III Dirac nodes which are formed by two bands crossing, one of which is dispersionless (flat) satisfying the condition for the formation of type-III Dirac cones.

A thorough investigation of the type-III crossings would require the imaging of the other side of the Dirac cone in the small segment $\mathrm{D} \Gamma$ [see inset of Fig. 5(c)] by scanning the photon energy in a range $>30 \mathrm{eV}$. However, from our calculations [inset of Fig. 5(c)], it can be inferred that the two bands are closely spaced between each other, differing by about $60 \mathrm{meV}$ only, as they both weakly disperse along $\mathrm{D} \Gamma$. Then, given that the energy resolution of our ARPES is limited by thermal excitations to around $100 \mathrm{meV}$ at room temperature, it would have been impossible to resolve their separation and their dispersion along the small segment $\mathrm{D} \Gamma$.
Therefore, our combined theoretical and experimental data limited to one part of the cone only, that is, the one along the DZ segment, provide evidence for a type-III Dirac node, while a full demonstration of this type of crossing is difficult due to band dispersion peculiarities and limited energy resolution in our room-temperature ARPES experiments.

Unlike the case of DSMs where a conduction and a valence band cross at a single point at or near the Fermi level, in SnTe, which is a narrow gap semiconductor, the bands involved are both valence bands crossing at $\sim 1.2 \mathrm{eV}$ below the Fermi level. By $p$-type doping it should be possible to change the chemical potential of SnTe to line up with the energy of the Dirac point. In such a case access of Dirac fermions in transport measurements is allowed to unveil the physics and exploit the potential benefits of a type-III Dirac cone in SnTe.

\section{ACKNOWLEDGMENTS}

This work was financially supported by the Laboratoire d'Alliances Nanosciences-Energies du futur (LANEF) Chair of Excellence program of Université Grenoble Alpes and Commissariat à l'Énergie Atomique et aux Énergies Alternatives (A.D.); the project MIS 5002567, cofinanced by Greece and the European Union (European Regional Development Fund) (P.T.); the Hellenic Foundation for Research and Innovation and the General Secretariat for Research and Technology, under Grant No. 435 (2D-TOP) (D.T. and E.X.); and the French state funds Grant No. ANR-10-LABX-51-01 (Labex LANEF du Programme d'Investissements d'Avenir) and Equipex Grant No. ANR-11-EQPX-0010 (G.R.). This work was also supported by computational time granted from the Greek Research and Technology Network in the National High-Performance Computing facility ARIS under projects TMD-WEYL and 2D-POLAR (pr002044 and pr005037_thin, respectively).

[9] N. P. Armitage, E. J. Mele, and A. Vishwanath, Rev. Mod. Phys. 90, 015001 (2018).

[10] G. E. Volovik, JETP Lett. 104, 645 (2016).

[11] G. E. Volovik and K. Zhang, Low Temp. Phys. 189, 276 (2017).

[12] H. Liu, J.-T. Sun, H. Huang, F. Liu, and S. Meng, arXiv:1809.00479v2.

[13] H. Huang, K.-H. Jin, and F. Liu, Phys. Rev. B 98, 121110 (2018).

[14] M. Milićević, G. Montambaux, T. Ozawa, O. Jamadi, B. Real, I. Sagnes, A. Lemaître, L. Le Gratiet, A. Harouri, J. Bloch, and A. Amo, Phys. Rev. X 9, 031010 (2019).

[15] T. Zhang, Y. Jiang, Z. Song, H. Huang, Y. He, Z. Fang, H. Weng, and C. Fang, Nature (London) 566, 475 (2019).

[16] M. G. Vergniory, L. Elcoro, C. Felser, N. Regnault, B. A. Bernevig, and Z. Wang, Nature (London) 566, 480 (2019).

[17] F. Tang, H. C. Po, A. Vishwanath, and X. Wan, Nature (London) 566, 486 (2019).

[18] A. Ishida, T. Tsuchiya, T. Yamada, D. Cao, S. Takaoka, M. Rahim, F. Felder, and H. Zogg, J. Appl. Phys. 107, 123708 (2010).

[8] A. K. Okazaki, S. Wiedmann, S. Pezzini, M. L. Peres, P. H. O. Rappl, and E. Abramof, Phys. Rev. B 98, 195136 (2018). 
[19] Y. Zhang, Z. Liu, B. Zhou, Y. Kim, L. Yang, H. Ryu, C. Hwang, Y. Chen, Z. Hussain, Z.-X. Shen, and S.-K. Mo, J. Electron Spectros. Relat. Phenomena 219, 35 (2017).

[20] A. A. Taskin, F. Yang, S. Sasaki, K. Segawa, and Y. Ando, Phys. Rev. B 89, 121302(R) (2014).

[21] T. Jiang, Y. Zang, H. Sun, X. Zheng, Y. Liu, Y. Gong, L. Fang, X. Cheng, and K. He, Adv. Opt. Mater. 5, 5 (2017).

[22] Y. Tanaka, T. Shoman, K. Nakayama, S. Souma, T. Sato, T. Takahashi, M. Novak, K. Segawa, and Y. Ando, Phys. Rev. B 88, 235126 (2013).

[23] Y. Shi, M. Wu, F. Zhang, and J. Feng, Phys. Rev. B 90, 235114 (2014).

[24] P. Tsipas, S. Kassavetis, D. Tsoutsou, E. Xenogiannopoulou, E. Golias, S. A. Giamini, C. Grazianetti, D. Chiappe, A. Molle, M. Fanciulli, and A. Dimoulas, Appl. Phys. Lett. 103, 251605 (2013).

[25] Joint Committee on Powder Diffraction Standards, International Centre for Diffraction Data, PDF-2 Release 2008, http: //www.icdd.com (accessed 24 Feb. 2017), card no. 65-0319.

[26] Z.-Y. Ye, H.-X. Deng, H.-Z. Wu, S.-S. Li, S.-H. Wei, and J.-W. Luo, npj Comput. Mater. 1, 15001 (2015).

[27] A. Béché, J. L. Rouvière, L. Clément, and J. M. Hartmann, Appl. Phys. Lett. 95, 123114 (2009).

[28] D. Cooper, N. Bernier, and J.-L. Rouvière, Nano Lett. 15, 5289 (2015).

[29] G. Kresse and J. Furthmüller, Comput. Mater. Sci. 6, 15 (1996).

[30] G. Kresse and J. Furthmüller, Phys. Rev. B 54, 11169 (1996).

[31] J. P. Perdew, K. Burke, and M. Ernzerhof, Phys. Rev. Lett. 77, 3865 (1996).

[32] H. Monkhorst and J. Pack, Phys. Rev. B 13, 5188 (1976).

[33] Z. Zhu, Y. Liu, Z.-M. Yu, S.-S. Wang, Y. X. Zhao, Y. Feng, X.-L. Sheng, and S. A. Yang, Phys. Rev. B 98, 125104 (2018).

[34] M. Yan, H. Huang, K. Zhang, E. Wang, W. Yao, K. Deng, G. Wan, H. Zhang, M. Arita, H. Yang, Z. Sun, H. Yao, Y. Wu, S. Fan, W. Duan, and S. Zhou, Nat. Commun. 8, 257 (2017).

[35] B. Bradlyn, L. Elcoro, J. Cano, M. G. Vergniory, Z. Wang, C. Felser, M. I. Aroyo, and B. A. Bernevig, Nature (London) 547, 298 (2017).

[36] M. G. Vergniory, L. Elcoro, Z. Wang, J. Cano, C. Felser, M. I. Aroyo, B. A. Bernevig, and B. Bradlyn, Phys. Rev. E 96, 023310 (2017).
[37] L. Elcoro, B. Bradlyn, Z. Wang, M. G. Vergniory, J. Cano, C. Felser, B. A. Bernevig, D. Orobengoa, G. de la Flor, and M. I. Aroyo, J. Appl. Crystallogr. 50, 1457 (2017).

[38] B.-J. Yang and N. Nagaosa, Nat. Commun. 5, 4898 (2014).

[39] L. Miao, Z. F. Wang, W. Ming, M.-Y. Yao, M. Wang, F. Yang, Y. R. Song, F. Zhu, A. V. Fedorov, Z. Sun, C. L. Gao, C. Liu, Q.-K. Xue, C.-X. Liu, F. Liu, D. Qian, and J.-F. Jia, PNAS 110, 2758 (2013).

[40] M. Iizumi, Y. Hamaguchi, K. F. Komatsubara, and Y. Kato, J. Phys. Soc. Jpn. 38, 443 (1975).

[41] K. V. Mitrofanov, A. V. Kolobov, P. Fons, M. Krbal, T. Shintani, J. Tominaga, and T. Uruga, Phys. Rev. B 90, 134101 (2014).

[42] L. Aggarwala, A. Banikb, S. Anand, U. V. Waghmare, K. Biswas, and G. Sheet, J. Materiomics 2, 196 (2016).

[43] Z. K. Liu, B. Zhou, Y. Zhang, Z. J. Wang, H. M. Weng, D. Prabhakaran, S.-K. Mo, Z. X. Shen, Z. Fang4, X. Dai, Z. Hussain, and Y. L. Chen, Science 343, 864 (2014).

[44] S.-Y. Xu, C. Liu, S. K. Kushwaha, R. Sankar, J. W. Krizan, I. Belopolski1, M. Neupane, G. Bian, N. Alidoust, T.-R. Chang, H.-T. Jeng, C.-Y. Huang, W.-F. Tsai, H. Lin, P. P. Shibayev, F.C. Chou, R. J. Cava, and M. Z. Hasan, Science 347, 294 (2015).

[45] M. Neupane, S.-Y. Xu, R. Sankar, N. Alidoust, G. Bian, C. Liu, I. Belopolski, T.-R. Chang, H.-T. Jeng, H. Lin, A. Bansil, F. Chou, and M. Z. Hasan, Nat. Commun. 5, 3786 (2014).

[46] Z. K. Liu, J. Jiang, B. Zhou, Z. J. Wang, Y. Zhang, H. M Weng, D. Prabhakaran, S-K. Mo, H. Peng, P. Dudin, T. Kim, M. Hoesch, Z. Fang, X. Dai, Z. X. Shen, D. L. Feng, Z. Hussain, and Y. L. Chen, Nat. Mater. 13, 677 (2014).

[47] S.-Y. Xu, I. Belopolski, N. Alidoust, M. Neupane, G. Bian, C. Zhang, R. Sankar, G. Chang, Z. Yuan, C.-C. Lee, S.-M. Huang, H. Zheng, J. Ma, D. S. Sanchez, B. Wang, A. Bansil, F. Chou, P. P. Shibayev, H. Lin, S. Jia, and M. Z. Hasan, Science 349, 613 (2015).

[48] L. X. Yang, Z. K. Liu, Y. Sun, H. Peng, H. F. Yang, T. Zhang, B. Zhou, Y. Zhang, Y. F. Guo, M. Rahn, D. Prabhakaran, Z. Hussain, S.-K. Mo, C. Felser, B. Yan, and Y. L. Chen, Nat. Phys. 11, 728 (2015)

[49] B. Q. Lv, N. Xu, H. M. Weng, J. Z. Ma, P. Richard, X. C. Huang, L. X. Zhao, G. F. Chen, C. E. Matt, F. Bisti, V. N. Strocov, J. Mesot, Z. Fang, X. Dai, T. Qian, M. Shi, and H. Ding, Nat. Phys. 11, 724 (2015).

[50] H. Huang, S. Zhou, and W. Duan, Phys. Rev. B 94, 121117(R) (2016). 